\title{
Enhancement of DNA Droplet Formation Ability in Liquid-Liquid Phase Separation by Introduction of Artificial Nucleobase
}

\author{
Ryu Tashiro*, Yuta Ito, Tomohiro Kitagawa \\ Faculty of Pharmaceutical Sciences, Suzuka University of Medical Science, 3500-3 Minamitamagaki-cho, Suzuka, Mie 513- \\ 8670, Japan
}

ABSTRACT: Controlling liquid-liquid phase separation (LLPS) of biomolecules is a challenge for understanding its biological phenomena and developing drugs for LLPS associated diseases. We found that substitution of DNA bases with 5-nitroindoles, known as universal bases, considerably improves the droplet formation ability of DNA. Our findings show the feasibility of introducing an artificial DNA nucleobase with a superior droplet formation ability, paving the way for the development of novel control systems for LLPS.

Numerous artificial nucleic acids have been developed and widely used to study structural changes of nucleic acids and enzymatic recognition associated with their biological functions. ${ }^{1-12}$ Liquid-liquid phase separation (LLPS) of biomacromolecules is a key physical phenomenon that underlies the formation of membrane-less compartments within cells. ${ }^{13-17}$ In recent years, it has been shown that nucleic acids have the ability to form droplets via LLPS and this ability is related to their cellular functions and several diseases. ${ }^{18-29}$

Controlling LLPS of DNA using artificial nucleobase is a novel challenge for expanding the possibilities of nucleic acid chemistry. This will be useful for LLPS related applications; it can serve as tools for investigating the functions of LLPS in nucleic acids and in the development of nucleic acid drugs for LLPS associated diseases. The LLPS of nucleic acids is affected by their sequence. ${ }^{18,19}$ We predicted that modification of the nucleobase moiety would allow the control of LLPS. However, the specificity of the sequences that induce LLPS remains unclear and the underlying mechanism is largely unknown. Therefore, preliminary information regarding modification of DNA for adjusting the efficiency of LLPS is lacking. As a first step, it is important to identify a modified DNA with an artificial nucleobase that can change the droplet formation ability. In this study, we built a system to evaluate the droplet formation efficiency of DNA. Using this system, we tested the effect of introducing artificial nucleobases on the efficiency of LLPS.

To simplify our experimental approach and minimize the effects of complicating factors such as the secondary structure of DNA, we elected to use short homo-oligomeric DNA as the parent molecule. However, the minimum length of singlestranded DNA that can form liquid droplets via LLPS was unknown. We therefore began by determining the shortest single-stranded homo-oligomeric DNA that could be used as the parent molecule in subsequent substitution experiments.

Polycationic biomolecules, such as peptides rich in lysine or arginine, mediate LLPS-based DNA and RNA droplet formation via electrostatic attraction towards the phosphate groups of nucleotides (Figure 1a). Such polycations have been use in previous studies as model systems for examining RNA and DNA coacervation. ${ }^{20-26}$ We, therefore, used 100-amino acid poly $(\mathrm{L})$ lysine as the polycation and observed the morphologies of the complexes that formed in mixtures of single-stranded homo-oligomeric DNA of various lengths (i.e., 4, 5, 6, or $8 \mathrm{nt}$ ) and poly(L)lysine.

When 6 or 8-nt DNA was mixed with poly $(L)$ lysine, the DNAs containing adenine, cytosine, or thymine, but not those containing guanine, formed liquid droplets (Figure 1b, c) that showed typical liquid characteristics such as a spherical shape and growth by fusion (Figure S1 in SI). Each sample contained $100 \mathrm{mM} \mathrm{NaCl}$. The salt concentration affected the droplet formation. In the system, droplet formation was inhibited by $\mathrm{NaCl}$ concentrations above $200 \mathrm{mM}$ (Figure S2). When 5-nt DNA was used, only the DNA containing adenine formed droplets. When 4-nt DNA was used, none of the DNAs formed droplets. Although none of the DNAs containing guanine formed droplets, they formed precipitates, presumably due to G-quadruplex structure formation. The turbidity measurement has been used to evaluate droplet formation. ${ }^{21}$ When the length of DNA was from 5 to $8 \mathrm{nt}$, the complex composed of adenine showed a higher turbidity than the other homo-oligomeric DNA molecules, indicating that the droplet formation ability of adenine was the highest among them (Figure 1d). When the DNA strand length was $5 \mathrm{nt}, 5^{\prime}-\mathrm{d}(\mathrm{C})_{5}-3^{\prime} /$ poly(L)Lysine complex also showed turbidity (Figure 1d). Considering the results of the microscopic observation (Figure 1b), the turbidity of the complex was not caused by droplets but by precipitates. Based on these findings, we concluded that the DNA containing five adenines (hereafter $A_{5}$ ) was the shortest DNA that was capable of forming liquid droplets in our system and we used this oligomer in the following experiments. It is also worth noting that the morphology (liquid droplet or precipitate) of the coacervates was dependent on the type and number of nucleobases in the DNA, indicating that nucleobases are an important determinant of coacervate morphology. 
We further hypothesized if substitution with a modified base, which is structurally similar to adenine but has different properties, could alter the ability of $A_{5}$ to form droplets. We replaced one or more of the adenines in $A_{5}$ with nucleoside 5nitroindole, which is the DNA analogue of a universal base that possesses a bicyclic aromatic moiety that can be involved in base stacking but cannot form hydrogen bonds between nucleobases. ${ }^{9-11}$ We then examined droplet formation using these substituted DNAs. To compare droplet formation ability, different concentrations of DNAs were used (Figure 2).
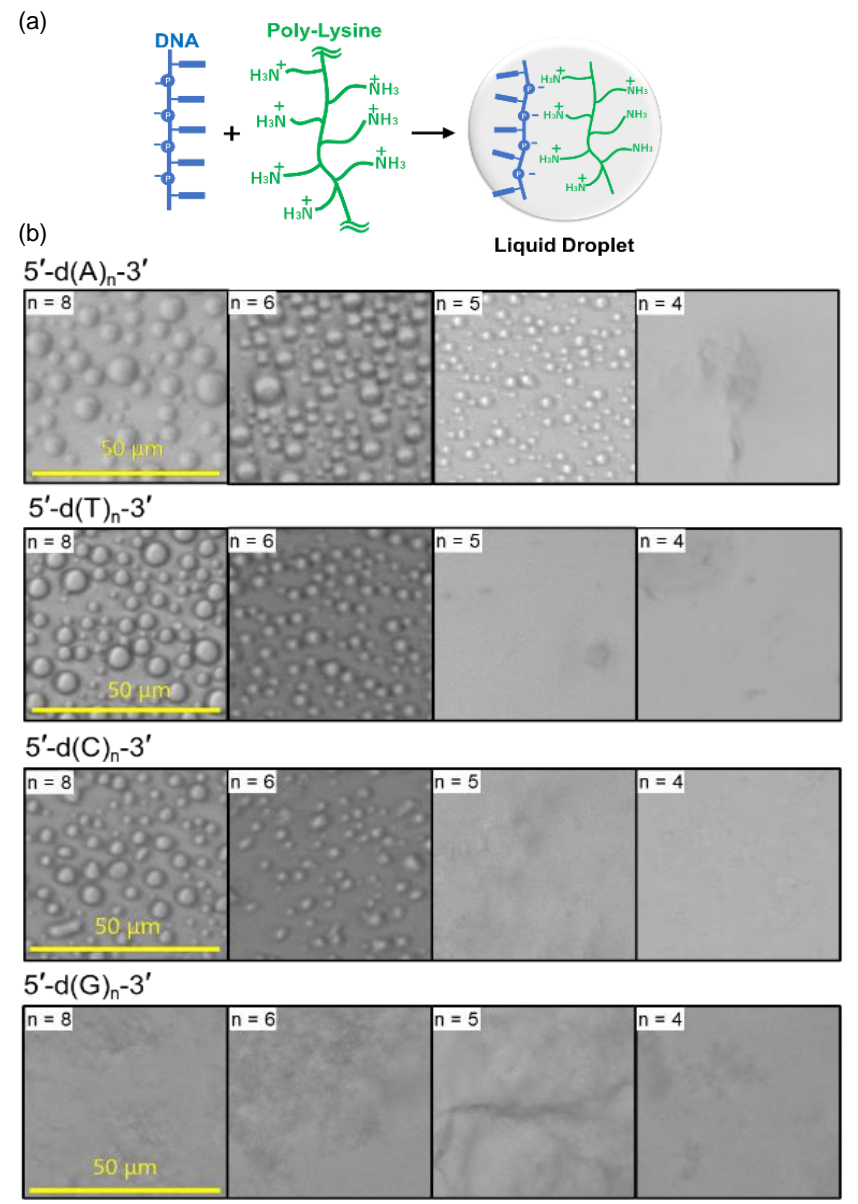

(c)

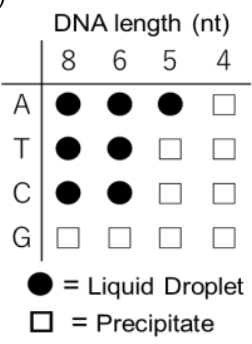

(d)

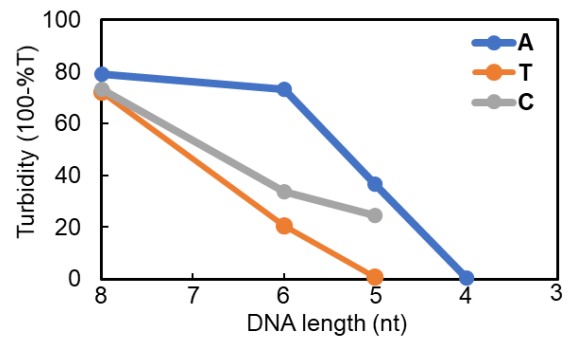

Figure 1. Effects of DNA length and identity of nucleobase on the morphology of DNA/poly(L)lysine complexes. (a) Schematic representation of poly(L)lysine-mediated liquid droplet formation. (b) Bright-field micrographs of the observed complexes (phosphate concentration from DNA, $5 \mathrm{mM}$; amine concentration from poly (L)lysine, $2.5 \mathrm{mM}$; charge ratio $[-] /[+]=2)$. (c) Summary of the observed complex morphologies. (d) Turbidity of the homo-oligomeric DNA (A, T, and C)/poly(L)Lysine complex. (a) A $_{5}: 5^{\prime}-$ d(AAAAA) $-3^{\prime}$
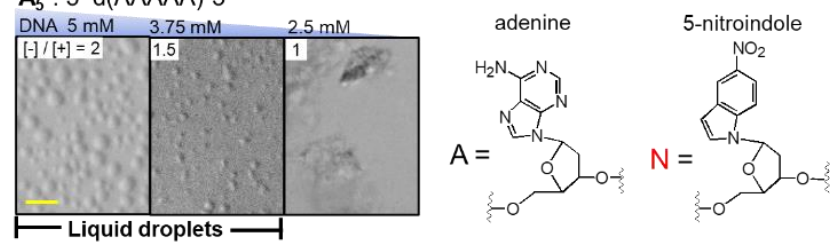

$\mathrm{N}_{1} \mathbf{A}_{4}: 5^{\prime}-\mathrm{d}(\mathrm{NAAAA})-3^{\prime}$

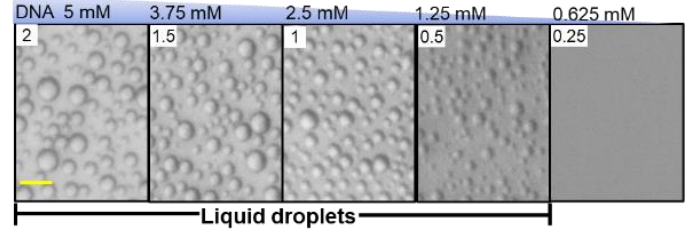

$\mathbf{N}_{2} \mathbf{A}_{3}: 5^{\prime}-\mathrm{d}\left(\right.$ NNAAA) $-3^{\prime}$

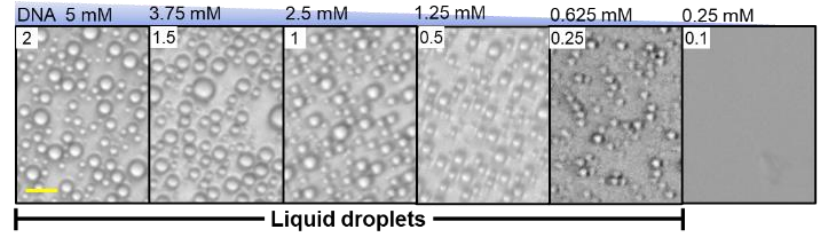

$\mathrm{N}_{3} \mathrm{~A}_{2}: 5^{\prime}-\mathrm{d}(\mathrm{NNNAA})-3^{\prime}$

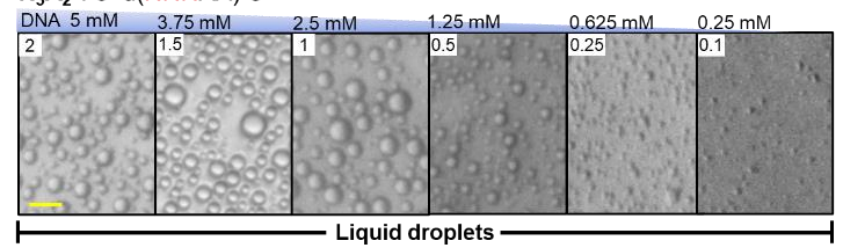

$\mathbf{N}_{4} \mathbf{A}_{1}: 5^{\prime}-\mathrm{d}(\mathrm{NNNNA})-3^{\prime}$

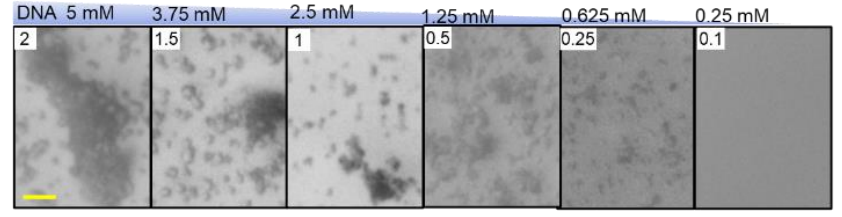

$\mathbf{N}_{5}: 5^{\prime}-\mathrm{d}(\mathrm{NNNNN})-3^{\prime}$

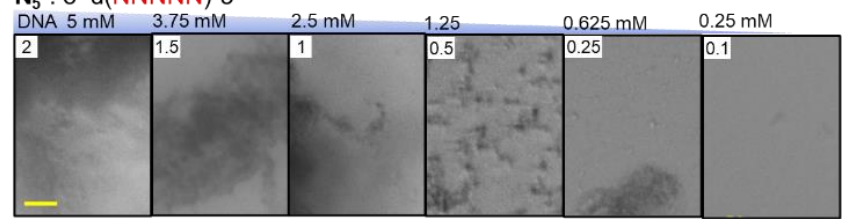

(b)

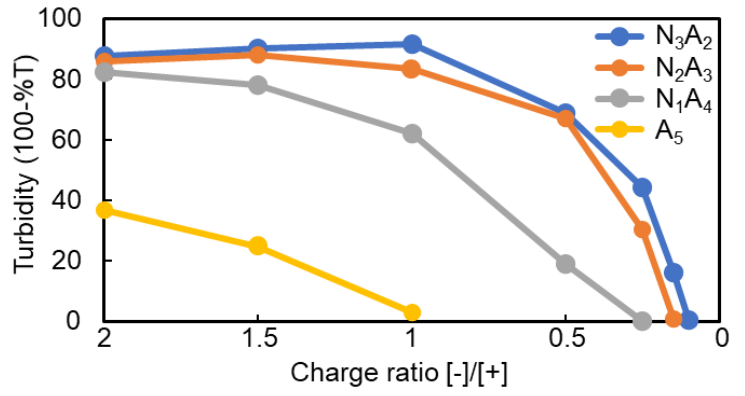

Figure 2. Effect of 5-nitroindole $(\mathrm{N})$ substitution on liquid droplet formation by a parent DNA molecule containing five adenines $\left(A_{5}\right)$. (a) Bright-field micrographs of mixtures of DNA (phosphate concentration, 5-0.25 mM) and poly(L)lysine (amine concentration, $2.5 \mathrm{mM}$ ). Inset numbers on images indicate the charge ratio. Scale bars, $10 \mu \mathrm{m}$. (b) Turbidity of the DNA $\left(A_{5}, N_{1} A_{4}, N_{2} A_{3}\right.$, and $\mathrm{N}_{3} \mathrm{~A}_{2}$ )/poly(L)Lysine complex. 
First, we observed the changes in the morphology of the $A_{5} /$ poly $(L)$ lysine complex with decreasing DNA concentration (phosphate concentration, 5-2.5 mM, change in charge ratio from 2 to 1). When the concentration of $A_{5}$ was decreased from 5 (charge ratio, 2) to $3.75 \mathrm{mM}$ (charge ratio, 1.5), droplet size was decreased. No droplets were formed at a DNA concentration of $2.5 \mathrm{mM}$ (charge ratio, 1). Under this charge ratio, precipitates were formed instead. This might be due to the presence of interactions between molecules, even though the liquid nature could not be maintained. To our surprise, when one to three of the adenines were substituted with 5-nitroindole, droplet formation was markedly enhanced. Whereas $A_{5}$ formed droplets only at a DNA concentration down to $3.75 \mathrm{mM}$, the DNA containing one substitution formed droplets down to $1.25 \mathrm{mM}$ (charge ratio, 0.5), the DNA containing two substitutions down to $0.625 \mathrm{mM}$ (charge ratio, 0.25 ), and the DNA containing three substitutions down to $0.25 \mathrm{mM}$ (charge ratio, 0.1 ). These results indicate that the DNA containing three substitutions had the highest droplet formation capability (i.e. the DNA formed droplets at the lowest DNA concentration). The results of turbidity measurement supported the enhancement effect of substitutions (Figure $2 b$ ). For example, when the charge ratio was 2 , the turbidity of droplets formed from the unsubstituted $A_{5}$ was about half that of the substituted DNA (Figure $2 b$ ). Interestingly, one 5 -nitroindole substitution $\left(\mathrm{N}_{1} \mathrm{~A}_{4}\right)$ significantly improved the droplet formation, and the effect was gradually saturated as the number of substitutions increased from one to three (Figure $2 b$ ). On the other hand, excessive substitutions inhibited the formation of droplets. When the number of substitutions was increased to four or five, precipitates rather than droplets were formed (Figure 2a). These results indicate that the efficiency of droplet formation is highly dependent on the number of substitutions with 5-nitroindole, and therefore, the efficiency is controllable by changing this number.

We propose several hypotheses regarding the effect of 5nitroindole on LLPS. Substitutions with 5-nitroindole reduce the number of functional groups involved in hydrogen bonding. We initially predicted that the enhancement effect of 5-nitroindole was due to a reduction in DNA interactions. However, substitution with abasic site analogs decreased the droplet formation ability (Figure S3). These results suggest that merely reducing interactions of DNA does not improve the droplet formation ability of DNA. Apparently, the mechanism of enhancement is not so simple. There may exist a balance between the interaction manners of nucleobases that mediates effective droplet formation. The substitution with 5-nitroindole changes the balance between hydrogen bonding and hydrophobic interactions. We predicted that this change in the balance of these interaction manners is one of the reasons for the improvement of droplet formation ability of DNA. As shown in Figure 2, the $\mathrm{N}_{3} \mathrm{~A}_{2}$ DNA in which $60 \%$ of the adenines were substituted with 5-nitroindol had the highest droplet formation capability. This result may provide a hint for the hypothesis. By focusing on this point, we aim to develop DNA with superior droplet formation ability in the future. The relevance of other weak interactions to droplet formation may also be found in this process. Although the underlying mechanism is still hypothetical at this point, in the development of modified DNA for LLPS will yield experimental validation of the hypothesis.
In summary, we found that LLPS of DNA is controllable by the introduction of artificial nucleobases; substitution of adenine with an appropriate number of 5-nitroindoles significantly increases the efficiency of droplet formation. In the process of selecting the DNA sequence to be modified, we found that homo-oligomeric DNA composed of adenine can form droplets at a shorter length than that composed of other bases. The present experiments with short DNA are important for a simpler understanding of the complex interactions that occur among huge nucleic acids in biological droplets. As a similar case, DNA melting experiments also measure short DNA that is not used by cells, but is an important indicator for discussing the stability of dsDNA.

The strong electrostatic attraction between the phosphate of the DNA and polycationic molecule has a significant effect on droplet formation. ${ }^{20-25}$ The interaction involving DNA nucleobases is very weak in comparison; nonetheless, it can control LLPS. We obtained modified DNAs with greater phase-separation ability than the parent DNA. Thus, these data provide a basis for the rational design of artificial nucleobases for the control of LLPS. Since natural DNA comprises only four types of nucleobases, research for LLPS of DNA has been limited. Since our base-modification strategy overcomes this limitation, we consider that it has the potential to provide new research directions.

\section{ASSOCIATED CONTENT}

\section{Supporting Information.}

Supporting Methods, Supporting Results (PDF).

\section{AUTHOR INFORMATION}

\section{Corresponding Author}

* Ryu Tashiro - Faculty of Pharmaceutical Sciences Suzuka University of Medical Science, 3500-3 Minamitamagaki-cho, Suzuka, Mie 513-8670 Japan.

Email: tashiro@suzuka-u.ac.jp

\section{Authors}

Yuta Ito - Faculty of Pharmaceutical Sciences Suzuka University of Medical Science, 3500-3 Minamitamagaki-cho, Suzuka, Mie 513-8670 Japan.

Tomohiro Kitagawa - Faculty of Pharmaceutical Sciences Suzuka University of Medical Science, 3500-3 Minamitamagaki-cho, Suzuka, Mie 513-8670 Japan.

\section{Notes}

The authors declare no competing financial interest.

\section{ACKNOWLEDGMENT}

We thank Prof. Seiji Komeda (Suzuka University of Medical Science) and Prof. Takanori Oyoshi (Shizuoka University) for helpful discussions.

\section{REFERENCES}

(1) Kool, E. T., Morales, J. C. and Guckian, K. M. (2000) Mimicking the structure and function of DNA: Insights into DNA stability and replication. Angew. Chem. Int. Ed. 39, 990-1009.

(2) Yadav, M., Kumar, R. and Krishnamurthy, R. (2020) Chemistry of abiotic nucleotide synthesis. Chem. Rev. 120, 4766-4805. 
(3) Shelton, J., Lu, X., Hollenbaugh, J. A., Cho, J. H., Amblard, F. and Schinazi. R. F. (2016) Metabolism, biochemical actions, and chemical synthesis of anticancer nucleosides, nucleotides, and base analogs. Chem. Rev. 116, 14379-14455.

(4) Kaur, H., Babu, B. R. and Maiti, S. (2007) Perspectives on chemistry and therapeutic applications of locked nucleic acid (LNA). Chem. Rev. 107, 4672-4697.

(5) Fantoni, N. Z., El-Sagheer, A. H. and Brown, T. (2016) A Hitchhiker's guide to click-chemistry with nucleic acids. Chem. Rev. 116, 422-518.

(6) Zhao, M., Steffen, F. D., Börner, R., Schaffer, M., F. Sigel, R. K. O. and Freisinger, E. (2018) Site-specific dual-color labeling of long RNAs for single-molecule spectroscopy. Nucleic Acids Res. 46. e13.

(7) Xu, Y., Tashiro, R. and Sugiyama, H. (2007) Photochemical Determination of Different DNA Structures. Nat. Protoc. 2, 78-87.

(8) Tashiro, R., Wang A. H. -J. and Sugiyama, H. (2006) Photoreactivation of DNA by an Archaeal Nucleoprotein Sso7d. Proc. Natl. Acad. Sci. U. S. A. 103, 16655-16659.

(9) Loakes, D. and Brown, D. M. (1994) 5-Nitroindole as an universal base analogue. Nucleic Acids Res. 22, 4039-4043.

(10) Bergstrom, D. E., Zhang, P. and Johnson, W. T. (1997) Comparison of the base pairing properties of a series of nitroazole nucleobase analogs in the oligodeoxyribonucleotide sequence 5'd(CGCXAATTYGCG)-3'. Nucleic Acids Res. 25, 1935-1942.

(11) Vallone, P. M. and Benight, A. S. (1999) Melting studies of short DNA hairpins containing the universal base 5-nitroindole. Nucleic Acids Res. 27, 3589-3596.

(12) de Jésus, K. P., Serre, L., Zelwer, C. and Castaing, B. (2005) Structural insights into abasic site for Fpg specific binding and catalysis: comparative high-resolution crystallographic studies of Fpg bound to various models of abasic site analogues-containing DNA. Nucleic Acids Res. 33, 5936-5944.

(13) Langdon, E. M., Qiu, Y., Niaki, A. G., McLaughlin, G. A.; Weidmann, C. A., Gerbich, T. M., Smith, J. A., Crutchley, J. M., Termini, C. M., Weeks, K. M., Myong, S. and Gladfelter, A. S. (2018) mRNA structure determines specificity of a polyQ-driven phase separation. Science 360, 922-927.

(14) Lu, Y., Wu, T., Gutman, O., Lu, H., Zhou, Q., Henis, Y. I. and Luo, K. (2020) Phase separation of TAZ compartmentalizes the transcription machinery to promote gene expression. Nat. Cell. Biol. 22, 453-464.

(15) Gallego, L. D., Schneider, M., Mittal, C., Romanauska, A., Carrillo, R. M. G., Schubert, T., Pugh, B. F. and Köhler A. (2020) Phase separation directs ubiquitination of gene-body nucleosomes. Nature 579, 592-597.

(16) Shin, Y. and Brangwynne, C. P. (2017) Liquid phase condensation in cell physiology and disease. Science 357, eaaf4382.

(17) Gabryelczyk, B., Cai, H., Shi, X., Sun, Y., Swinkels, P. J. M., Salentinig, S., Pervushin, K. and Miserez. A. (2019) Hydrogen bond guidance and aromatic stacking drive liquid-liquid phase separation of intrinsically disordered histidine-rich peptides. Nat. Commun. 10, 5465.

(18) Jain, A. and Vale, R. D. (2017) RNA phase transitions in repeat expansion disorders. Nature 546, 243-247.

(19) Zhang, Y., Yang, M., Duncan, S., Yang, X., Abdelhamid, M. A. S., Huang, L., Zhang, H., Benfey, P. N., Waller Z. A. E. and Ding, Y. (2019) G-quadruplex structures trigger RNA phase separation. Nucleic. Acids. Res. 47, 11746-11754.

(20) Vieregg, J. R., M. Lueckheide, A. B., Marciel, L., Leon, A. J., Bologna, Rivera, J. R. and Tirrell. M. V. (2018) Oligonucleotide-Peptide Complexes: Phase Control by Hybridization. J. Am. Chem. Soc. 140, 16321638.

(21) Aumiller, Jr., W. M.; Cakmak, F. P., Davis, B. W. and Keating C. D. (2016) RNA-Based coacervates as a model for membraneless organelles: formation, properties, and interfacial liposome assembly. Langmuir 32, 10042-10053.

(22) Aumiller, Jr., W. M. and Keating, C. D. (2016) Phosphorylationmediated RNA/peptide complex coacervation as a model for intracellular liquid organelles. Nat. Chem. 8, 129-137.
(23) Boeynaems, S., Holehouse, A. S., Weinhardt, V., Kovacs, D., Lindt, J. V., Larabell, C., Bosch, L. V. D., Das, R., Tompa, P. S., Pappu, R. V. and Gitler, A. D. (2019) Spontaneous driving forces give rise to protein-RNA condensates with coexisting phases and complex material properties. Proc. Natl. Acad. Sci. U. S. A. 116. 7889-7898.

(24) Onuchic, P. L., Milin, A. N., Alshareedah, I., Deniz, A. A. and Banerjee, P. R. (2019) Divalent cations can control a switch-like behavior in heterotypic and homotypic RNA coacervates. Sci. Rep. 9, 12161. (25) Lu, T. and Spruijt, E. (2020) Multiphase Complex Coacervate Droplets. J. Am. Chem. Soc. 142, 2905-2914.

(26) Fischer, R. S. and Elbaum-Garfinkle, S. E. (2020) Tunable multiphase dynamics of arginine and lysine liquid condensates. Nat. Commun. 11, 4628.

(27) Roden, C. and Gladfelter, A. S. (2020) RNA contributions to the form and function of biomolecular condensates. Nat. Rev. Mol. Cell. Biol. https://doi.org/10.1038/s41580-020-0264-6.

(28) Treeck, B. V., Protter, D. S. W., Matheny, T., Khong, A., Link, C. D. and Parker R. (2018) RNA self-assembly contributes to stress granule formation and defining the stress granule transcriptome. Proc. Natl. Acad. Sci. U. S. A. 115, 2734-2739.

(29) Treeck, B. V. and Parker R. (2018) Emerging Roles for Intermolecular RNA-RNA Interactions in RNP Assemblies. Cell 174, 791-802. 


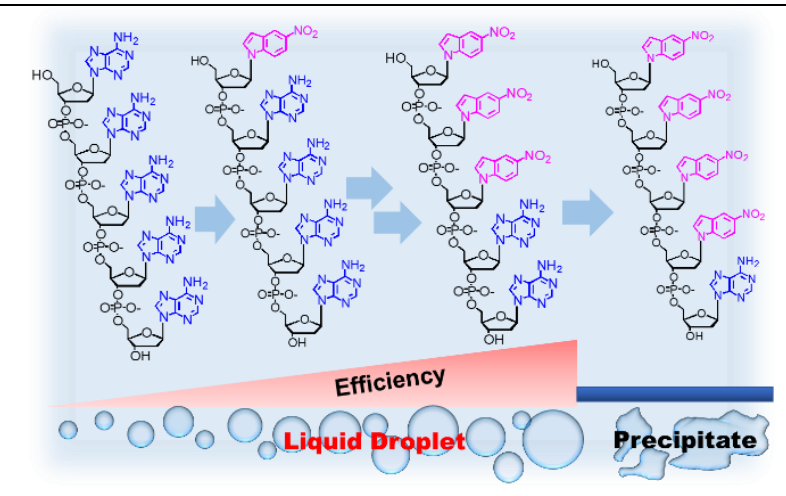

